\title{
A Cooperative Approach to Queue Allocation of Indivisible Objects
}

\author{
Herbert Hamers $^{\mathrm{a}} \quad$ Flip Klijn $^{\mathrm{b}}$ Marco Slikker $^{\mathrm{c}} \quad$ Bas van Velzen $^{\mathrm{d}}$
}

September 2004

\begin{abstract}
We consider the allocation of a finite number of indivisible objects to the same number of agents according to an exogenously given queue. We assume that the agents collaborate in order to achieve an efficient outcome for society. We allow for side-payments and provide a method for obtaining stable outcomes.
\end{abstract}

Keywords: indivisible objects, queue, cooperative game theory JEL classification: C71, D61, D70

\section{Introduction}

Many housing associations use waiting lists to allocate houses to tenants. Typically, the tenant on top of the waiting list is assigned his top choice, the tenant ordered second is assigned his top choice among the remaining houses, etc. A major reason why this mechanism is considered not very desirable is that the outcome of the procedure might not be efficient for society. In particular, by collaboration the total group of tenants might be able to achieve a higher utility.

A situation where a finite number of indivisible objects need to be allocated to the same number of individuals with respect to some queue is studied in [5]. To be more precise, [5] discusses a situation with a finite number of indivisible objects, the same number of individuals, and an exogenously given queue. Subsequently, an allocation method is proposed and it is shown that it satisfies certain desirable properties.

The difference between our model and that of [5] is that we assume that the preferences of the agents over the set of objects are expressed in monetary units. This implies that the allocation

\footnotetext{
${ }^{a}$ CentER and Department of Econometrics and OR, Tilburg University, P.O. Box 90153, 5000 LE Tilburg, The Netherlands. E-mail: H.J.M.Hamers@uvt.nl.

b Institut d'Anàlisi Econòmica (CSIC), Campus UAB, 08193 Bellaterra (Barcelona), Spain. E-mail: fklijn@pareto.uab.es. F. Klijn's research is supported by a Ramón y Cajal contract of the Spanish Ministerio de Ciencia y Tecnología. His work is also partially supported by Research Grant BEC2002-02130 from the Spanish Ministerio de Ciencia y Tecnología and by the Barcelona Economics Program of CREA.

${ }^{\mathrm{c}}$ Department of Technology Management, Technische Universiteit Eindhoven, P.O. Box 513, 5600 MB Eindhoven, The Netherlands. E-mail: M.Slikker@tm.tue.nl.

${ }^{\mathrm{d}}$ Corresponding author. CentER and Department of Econometrics and OR, Tilburg University, P.O. Box 90153, 5000 LE Tilburg, The Netherlands. E-mail: svelzen@uvt.nl.
} 
proposed by [5] might not be efficient for society. Only by collaborating the agents will be able to reach a society-efficient allocation. Because of this collaboration individual agents might not be satisfied with the final assignment of the objects. We assume that these agents are compensated by means of side-payments. Our main result is that the society-efficient assignment is supported by side-payments that guarantee stability, i.e., no coalition has an incentive to split off from the grand coalition.

Another well-known model with indivisible objects is the housing market of [3]. This housing market considers a finite number of agents, each initially possessing an object (house). The agents have preferences over the set of objects. It is shown that core allocations exist for this model. In [6] the model of [3] is adapted by assuming that the preferences of the agents can be expressed by monetary units. In this way the class of permutation games is introduced and the non-emptiness of the core is shown. Hence, our work parallels the approach to the housing market.

The remainder of this note is organized as follows. In Section 2 we briefly discuss several concepts from cooperative and non-cooperative game theory. In Section 3 we present our model and associate with each object allocation situation a cooperative game. In Section 4 we first provide a method to obtain stable allocations by using assignment games ( $c f$. [4]). Next, we show that when agents have a common rank order over the objects our cooperative game coincides with a related permutation game.

\section{Game theoretical preliminaries}

In this section we shortly introduce some game theoretical concepts. First we recall some notions from cooperative game theory. The section ends with a brief description of extensive form games.

A cooperative game is a pair $(N, v)$ where $N=\{1, \ldots, n\}$ is a finite set of players and $v$, the characteristic function, is a map $v: 2^{N} \rightarrow \mathbb{R}$ with $v(\emptyset)=0$. The map $v$ assigns to each subset $S \subseteq N$, called a coalition, a real number $v(S)$, called the worth of $S$. The core of a game $(N, v)$ is the set $C(v)=\left\{x \in \mathbb{R}^{N}: \sum_{i \in S} x_{i} \geq v(S)\right.$ for every $S \subseteq N$ and $\left.\sum_{i \in N} x_{i}=v(N)\right\}$. Intuitively, the core is the set of efficient payoff vectors for which no coalition has an incentive to split off from the grand coalition. The core can be empty.

A bipartite matching situation $(N, M, U)$ consists of two disjoint finite sets of agents $N=$ $\{1, \ldots, n\}, M=\{1, \ldots, m\}$, and an $n \times m$-matrix $U$. If agents $i \in N$ and $j \in M$ collaborate they achieve a utility of $U_{i j} \in \mathbb{R}$. This matching situation was first modelled as a cooperative game in [4], in the following way. Let $S \subseteq N$ and $T \subseteq M$. A matching $\mu$ for $S \cup T$ is a map from $S \cup T$ onto itself of order two (that is, $\mu(\mu(i))=i$ for all $i \in S \cup T$ ) such that for all $i \in S$ with $\mu(i) \neq i$ it holds that $\mu(i) \in T$, and for all $j \in T$ with $\mu(j) \neq j$ it holds that $\mu(j) \in S$. We write $(i, j) \in \mu$ if $\mu(i)=j$ for $i \in S$ and $j \in T$. Let $\mathcal{M}(S, T)$ denote the set of all matchings for coalition $S \cup T$. The assignment game $\left(N \cup M, v_{A}\right)$ is defined by $v_{A}(S \cup T)=\max \left\{\sum_{(i, j) \in \mu} U_{i j}: \mu \in \mathcal{M}(S, T)\right\}$ for all $S \subseteq N, T \subseteq M$. That is, the worth of a coalition is obtained by maximizing the sum of utilities over the set of matchings for this coalition. A matching that maximizes the sum of utilities is called optimal for the coalition. An optimal matching for $N \cup M$ is simply called optimal. It is well-known that assignment games have a non-empty core (cf. [4]). In particular, let $\mu$ be an optimal matching and let $x=(u, v) \in \mathbb{R}^{N} \times \mathbb{R}^{M}$. Then it holds that $x \in C\left(v_{A}\right)$ if and only if $u_{i}+v_{j}=U_{i j}$ for each $(i, j) \in \mu, u_{i}+v_{j} \geq U_{i j}$ for each $i \in N, j \in M$, and $x_{k} \geq 0$ for each $k \in N \cup M$. 
A permutation situation $(N, M, U)$ consists of a finite set of agents $N=\{1, \ldots, n\}$, a finite set of objects $M=\{1, \ldots, n\}$ and an $n \times n$-matrix $U$. Each agent $i \in N$ initially possesses object $i \in M$. The utility that agent $i \in N$ receives from the consumption of object $j \in M$ is given by $U_{i j} \in \mathbb{R}$. By reallocating their initially owned objects the agents can possibly achieve a higher utility. Permutation situations can be modelled as cooperative games in the following way. A reallocation of the objects of coalition $S \subseteq N$ among the members of $S$ can be expressed by a bijection $\pi_{S}: S \rightarrow O(S)$, where $O(S)$ denotes the set of objects initially owned by coalition $S$. Let $\Pi(S, O(S))$ denote the set of all bijections from $S$ to $O(S) .{ }^{1}$ The permutation game $\left(N, v_{P}\right)$ is defined by $v_{P}(S)=\max \left\{\sum_{i \in S} U_{i \pi_{S}(i)}: \pi_{S} \in \Pi(S, O(S))\right\}$ for all $S \subseteq N$. That is, the worth of a coalition is the maximum utility it can achieve by reallocating their initially owned objects among its members.

Permutation games were studied first in [6]. In that paper a link was established between the cores of assignment games and permutation games. It was shown that each core element of an assignment game gives rise to a core element of a related permutation game. In [2] it was shown that all core elements of a permutation game can be obtained from the core of some associated assignment game.

To conclude this section we shortly introduce extensive form games. ${ }^{2}$ We first remark that we only consider extensive form games with perfect information, i.e., extensive form games with information sets of cardinality one and without chance nodes. An extensive form game is a 4-tuple $(P, T, C, u)$, where $P$ is a finite set of players, $T$ is a rooted tree with non-terminal node set $V_{1}$ and terminal node set $V_{2}, C: V_{1} \rightarrow P$ is a control function, and $u: V_{2} \rightarrow \mathbb{R}^{P}$ is a function expressing the utility that each player receives at each terminal node. For each $i \in P$ let $c_{i} \subseteq V_{1}$ be the set of nodes controlled by $i$, i.e., $c_{i}=\left\{v \in V_{1}: C(v)=i\right\}$. A strategy of player $i \in P$ is a map $y_{i}: c_{i} \rightarrow V_{1} \cup V_{2}$ such that $\left(v, y_{i}(v)\right)$ is an arc in $T$ for all $v \in c_{i}$. So a strategy for player $i$ describes at each node controlled by player $i$ the direction in which the game proceeds. The set of all strategies of player $i$ is denoted by $\Sigma_{i}$. It is obvious that each strategy profile $\left(y_{i}\right)_{i \in P}$ leads to a unique terminal node. Hence we can write, with slight abuse of notation, the utility function $u$ as a function of strategy profiles, i.e., $u\left(\left(y_{i}\right)_{i \in P}\right)=u(v)$ if $v \in V_{2}$ is the terminal node reached by $\left(y_{i}\right)_{i \in P}$. We say that $y_{i} \in \Sigma_{i}$ is a best reply for player $i$ against $y_{-i}=\left(y_{j}\right)_{j \in P \backslash\{i\}} \in\left(\Sigma_{j}\right)_{j \in P \backslash\{i\}}$ if it holds that $u_{i}\left(y_{-i}, y_{i}\right) \geq u_{i}\left(y_{-i}, z_{i}\right)$ for all $z_{i} \in \Sigma_{i}$. In other words, a player's strategy is a best reply against some strategy profile of the other players if he cannot be strictly better off by unilaterally deviating from this strategy.

\section{The object allocation situation and game}

In this section we introduce our object allocation situation and a corresponding cooperative game.

An object allocation situation is a 4 -tuple $\left(N, M, U, \sigma_{0}\right)$. Here $N=\{1, \ldots, n\}$ is a set of agents, $M=\{1, \ldots, m\}$ is a set of indivisible objects, $U$ is a non-negative $n \times m$-matrix that gives the utility of each object for each agent, and $\sigma_{0}$ is an initial order. We assume that there are as many agents as objects, i.e., $n=m .^{3}$ The initial order should be interpreted as the order in which the

\footnotetext{
${ }^{1}$ We denote the set of bijections from a set $A$ to a set $B$ by $\Pi(A, B)$.

${ }^{2}$ For a full description of extensive form games, see, e.g, [1].

${ }^{3}$ The situation where $m<n$ is captured by introducing worthless null objects.
} 
agents may choose from the set of objects, i.e., agent $\sigma_{0}(1)$ has the first choice, agent $\sigma_{0}(2)$ the second, etc. Without loss of generality, let $\sigma_{0}(i)=i$ for all $i \in\{1, \ldots, n\}$.

Let $\left(N, M, U, \sigma_{0}\right)$ be an object allocation situation. We will analyze this situation using cooperative game theory. At our cooperative game we define the worth $v(S)$ of a coalition $S \subseteq N$ as the maximum total utility it can guarantee itself without any help from $N \backslash S$. This utility can be determined in two stages. In the first stage, all players sequentially choose an object, respecting $\sigma_{0}$. In the second stage, the members of $S$ reallocate the chosen objects among themselves to reach coalitional efficiency. Obviously, the outcome of this reallocation depends on the objects chosen by the members of $S$, and therefore also on the objects chosen by the members of $N \backslash S$.

In order to describe the value $v(S)$ of a coalition $S \subseteq N$, we define an (auxiliary) extensive form game $\left(\{S, N \backslash S\}, T, C^{S}, u^{S}\right)$ with player set $\{S, N \backslash S\}$. We first describe the rooted tree $T$. Let $1 \leq k \leq m$. The set of injective maps from $\{1, \ldots, k\}$ to $M$ is denoted by $\mathcal{S}_{k}$. A map $\pi \in \mathcal{S}_{k}$ is interpreted as a situation where object $\pi(i)$ is chosen by agent $i$ for each $1 \leq i \leq k$. Similarly, we define $\mathcal{S}_{0}$ as the situation where none of the objects is chosen yet. Let $T$ be the rooted tree with node set $\cup_{0 \leq k \leq m} \mathcal{S}_{k}$ and root $\mathcal{S}_{0}$. There is an arc between $\pi \in \mathcal{S}_{k}$ and $\tau \in \mathcal{S}_{k+1}$ with $0 \leq k \leq m-1$, if and only if $\pi(i)=\tau(i)$ for all $1 \leq i \leq k$. That is, there is an arc between $\pi$ and $\tau$ if $\pi$ can be extended to $\tau$ by assigning an appropriate object to player $k+1$. So, $V_{1}=\cup_{0 \leq k \leq m-1} \mathcal{S}_{k}$ and $V_{2}=\mathcal{S}_{m}$ are the sets of non-terminal and terminal nodes, respectively.

We define the control function $C^{S}: \cup_{0 \leq k \leq m-1} \mathcal{S}_{k} \rightarrow\{S, N \backslash S\}$ as follows. Let $\pi \in \mathcal{S}_{k}$ for some $0 \leq k \leq m-1$. Then we define $C^{S}(\pi)=S$ if and only if $k+1 \in S$. So coalition $S$ controls the nodes at which one of its members is to choose an object. Let $\Sigma_{S}$ and $\Sigma_{N \backslash S}$ be the set of all possible strategies of players $S$ and $N \backslash S$, respectively.

Finally, we describe the utility function $u^{S}: \Sigma_{S} \times \Sigma_{N \backslash S} \rightarrow \mathbb{R}^{\{S, N \backslash S\}}$. Let $y=\left(y_{S}, y_{N \backslash S}\right) \in \Sigma_{S} \times$ $\Sigma_{N \backslash S}$. Let $\tau \in \mathcal{S}_{m}$ be the terminal node reached by strategy profile $y$, and let $H_{S}(\tau)=\{\tau(i): i \in S\}$ be the corresponding set of objects obtained by $S$. Now define $u_{S}^{S}(y)=\max \left\{\sum_{i \in S} U_{i \pi(i)}: \pi \in\right.$ $\left.\Pi\left(S, H_{S}(\tau)\right)\right\}$, and $u_{N \backslash S}^{S}(y)=-u_{S}^{S}(y)$. So, the payoff of $S$ at terminal node $\tau \in \mathcal{S}_{m}$ is the maximum utility $S$ obtains after reallocating the initially chosen objects and the payoff for $N \backslash S$ is just the opposite of the payoff of $S$. Hence, $N \backslash S$ maximizes its payoff at the extensive form game by minimizing the payoff of $S$.

Now we define the object allocation game $(N, v)$ by

$$
v(S)=\max _{y_{S} \in \Sigma_{S}} \min _{y_{N \backslash S} \in \Sigma_{N \backslash S}} u_{S}^{S}(y) \text { for all } S \subseteq N .
$$

Note that $v(S)$ is precisely the maximum utility coalition $S$ can guarantee itself, i.e., without any help from $N \backslash S$. Also, notice that $v(N)=v_{A}(N \cup M)$, where $\left(N \cup M, v_{A}\right)$ is the assignment game corresponding to the bipartite matching situation $(N, M, U)$.

We illustrate the object allocation game in the following example.

Example 3.1 Let $N=\{1,2,3\}, M=\{A, B, C\}$, and $U=\left(\begin{array}{lll}3 & 6 & 2 \\ 4 & 5 & 3 \\ 5 & 3 & 0\end{array}\right)$. The object allocation 


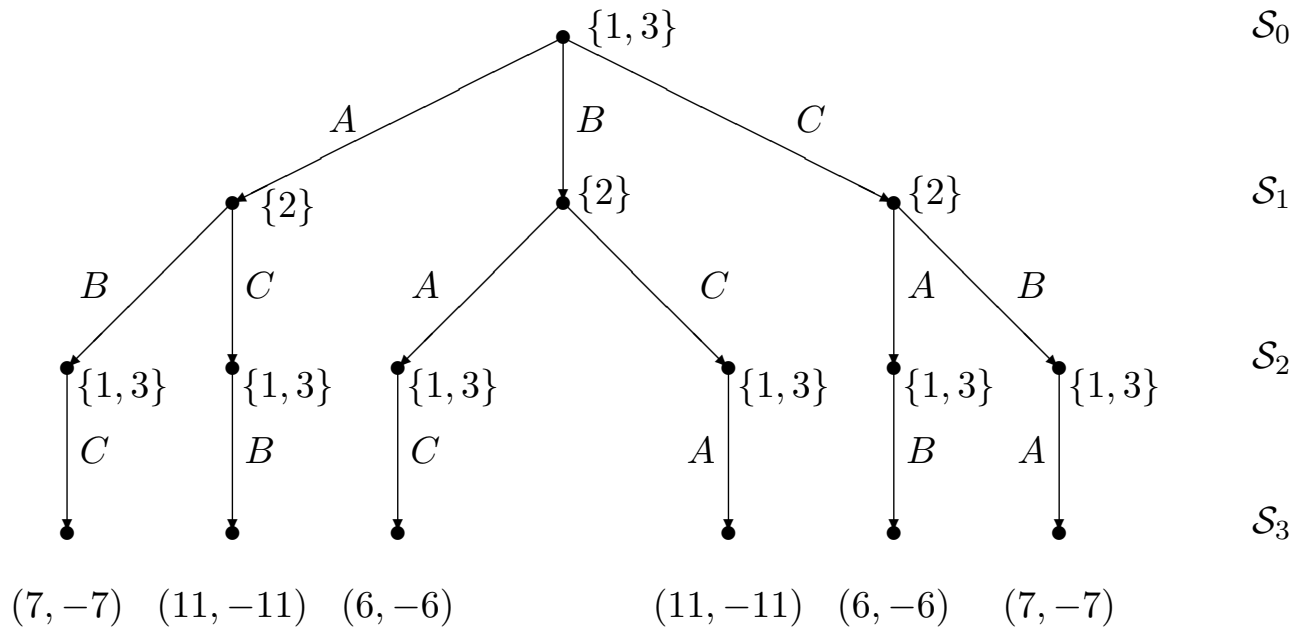

Figure 1: The extensive form game $\left(\{\{1,3\},\{2\}\}, T, C^{\{1,3\}}, u^{\{1,3\}}\right)$.

game $(N, v)$ is given by

\begin{tabular}{|c|ccccccc|}
\hline$S$ & $\{1\}$ & $\{2\}$ & $\{3\}$ & $\{1,2\}$ & $\{1,3\}$ & $\{2,3\}$ & $\{1,2,3\}$ \\
\hline$v(S)$ & 6 & 4 & 0 & 10 & 7 & 6 & 14 \\
\hline
\end{tabular}

To see for instance why $v(\{1,3\})=7$ consider the extensive form game $(\{\{1,3\},\{2\}\}, T$, $\left.C^{\{1,3\}}, u^{\{1,3\}}\right)$ which is depicted in Figure 1. If coalition $\{1,3\}$ chooses object A as a first choice, then the utility it will achieve is equal to 7 , since player $\{2\}$ will choose object $B$ in order to maximize its own payoff at the extensive form game. If coalition $\{1,3\}$ chooses object $\mathrm{B}$ or $\mathrm{C}$ first, then coalition $\{2\}$ will obviously maximize its payoff at the extensive form game by choosing object A. This leads to a utility of 6 for coalition $\{1,3\}$. Hence, coalition $\{1,3\}$ can guarantee itself a payoff of 7 by first choosing object $A$. We conclude that $v(\{1,3\})=7$.

\section{Results}

In this section we first show that the core of object allocation games is non-empty. In fact, we provide a method to obtain core elements by using core elements from a related assignment game. Furthermore, we show that for a special class of utility profiles the object allocation game coincides with a corresponding permutation game.

The following theorem shows the non-emptiness of the core of object allocation games.

Theorem 4.1 Let $\left(N, M, U, \sigma_{0}\right)$ be an object allocation problem and let $(N, v)$ be its corresponding game. Let $(N, M, U)$ be the corresponding bipartite matching problem and let $\left(N \cup M, v_{A}\right)$ be the corresponding assignment game. Let $(u, w) \in C\left(v_{A}\right)$ and let $\tau:\{1, \ldots, m\} \rightarrow M$ be a bijection such that $w_{\tau(1)} \geq \ldots \geq w_{\tau(m)}$. Define $x_{i}=u_{i}+w_{\tau(i)}$ for all $i \in N$. Then, $x \in C(v)$. 
Proof: By definition of $x, \sum_{i \in N} x_{i}=v_{A}(N \cup M)$. Since $v_{A}(N \cup M)=v(N), \sum_{i \in N} x_{i}=v(N)$.

It remains to show stability. Let $S \subseteq N$ and consider the extensive form game $\left(\{S, N \backslash S\}, T, C^{S}\right.$, $u^{S}$ ). Consider the following (possibly non-optimal) strategy $z_{N \backslash S} \in \Sigma_{N \backslash S}$ for player $N \backslash S$ : "always pick the object with highest $w_{i}$ that is still available." More precisely, let $z_{N \backslash S} \in \Sigma_{N \backslash S}$ be such that $z_{N \backslash S}(\sigma)=\tau$ for each $\sigma \in \mathcal{S}_{k}, k+1 \in N \backslash S$, and $\tau \in \mathcal{S}_{k+1}$ with $w_{\tau(k+1)} \geq w_{j}$ for all $j \in M \backslash\{\sigma(1), \ldots, \sigma(k)\}$.

Now if player $S$ would use a similar strategy in the strategic form game as player $N \backslash S$, i.e., also "always pick the highest $w_{i}$ that remains," then player $S$ would acquire $\{\tau(i): i \in S\}$ as its set of objects. If player $S$ uses a different strategy, then, given player $N \backslash S$ 's strategy $z_{N \backslash S}$, it would obtain a set of objects $A$ with lower $w_{i}$-values. Formally,

$$
\sum_{a \in A} w_{a} \leq \sum_{i \in S} w_{\tau(i)}
$$

In particular, let player $S$ play a best reply against strategy $z_{N \backslash S}$. Let $A^{*}$ be the set of objects acquired by $S$. Let $\pi: S \rightarrow A^{*}$ be the optimal reallocation of the obtained objects. From (1) it follows that

$$
\sum_{i \in S} w_{\pi(i)}=\sum_{a \in A^{*}} w_{a} \leq \sum_{i \in S} w_{\tau(i)} .
$$

Hence,

$$
\sum_{i \in S} x_{i}=\sum_{i \in S} u_{i}+\sum_{i \in S} w_{\tau(i)} \geq \sum_{i \in S} u_{i}+\sum_{i \in S} w_{\pi(i)} \geq v_{A}(S \cup\{\pi(i): i \in S\})=\sum_{i \in S} U_{i \pi(i)} .
$$

The first inequality is due to $(2)$. The second inequality is satisfied because $(u, w) \in C\left(v_{A}\right)$. The last equality is satisfied since the matching $\{(i, \pi(i)): i \in S\}$ is an optimal reallocation, and hence optimal for coalition $S \cup\{\pi(i): i \in S\}$ at the assignment game $\left(N, v_{A}\right)$.

From the definition of the game $(N, v)$ it follows that

$$
\sum_{i \in S} U_{i \pi(i)}=\max _{y_{S} \in \Sigma_{S}} u_{S}^{S}\left(y_{S}, z_{N \backslash S}\right) \geq \max _{y_{S} \in \Sigma_{S}} \min _{y_{N \backslash S} \in \Sigma_{N \backslash S}} u_{S}^{S}(y)=v(S) .
$$

Now the theorem follows immediately from (3) and (4).

The next example illustrates Theorem 4.1. Moreover, it shows that in general not all core elements of the object allocation game can be obtained via the technique of Theorem 4.1.

Example 4.1 Let $\left(N, M, U, \sigma_{0}\right)$ be the object allocation situation from Example 3.1, and $(N, v)$ the corresponding game. Consider the corresponding bipartite matching situation $(N, M, U)$ and assignment game $\left(N \cup M, v_{A}\right)$. Note that $(u, w) \in C\left(v_{A}\right)$ with $u=(2,3,3)$ and $w=\left(w_{A}, w_{B}, w_{C}\right)=$ $(2,4,0)$. Clearly $w_{B} \geq w_{A} \geq w_{C}$. Now let $x_{1}=u_{1}+w_{B}=6, x_{2}=u_{2}+w_{A}=5$, and $x_{3}=u_{3}+w_{C}=$ 3. From Theorem 4.1 it follows that $x=(6,5,3) \in C(v)$.

We will now show that not each element of $C(v)$ is achievable by the method of Theorem 4.1. Consider $y=(8,4,2) \in C(v)$. Suppose that $\left(u^{\prime}, w^{\prime}\right) \in C\left(v_{A}\right)$ is such that $u_{1}^{\prime}+w_{\tau(1)}^{\prime}=8$, $u_{2}^{\prime}+w_{\tau(2)}^{\prime}=4$, and $u_{3}^{\prime}+w_{\tau(3)}^{\prime}=2$ where $\tau:\{1,2,3\} \rightarrow M$ is a bijection with $w_{\tau(1)}^{\prime} \geq w_{\tau(2)}^{\prime} \geq w_{\tau(3)}^{\prime}$. 
First note that $(N, M, U)$ has a unique optimal matching $\mu=\{(1, B),(2, C),(3, A)\}$. So, since $\left(u^{\prime}, w^{\prime}\right) \in C\left(v_{A}\right)$, it holds that $u_{1}^{\prime}+w_{B}^{\prime}=U_{1 B}=6, u_{2}^{\prime}+w_{C}^{\prime}=U_{2 C}=3$, and $u_{3}^{\prime}+w_{A}^{\prime}=U_{3 A}=5$. Since $u_{2}^{\prime}+w_{\tau(2)}^{\prime}=4$ it follows that $w_{\tau(2)}^{\prime}>w_{C}^{\prime}$. So, $w_{\tau(1)}^{\prime} \geq w_{\tau(2)}^{\prime}>w_{C}^{\prime}$. Hence, $\tau(3)=C$. Because $u_{1}^{\prime}+w_{\tau(1)}^{\prime}=8$ it follows that $w_{\tau(1)}^{\prime}>w_{B}^{\prime}$, and thus that $\tau(1) \neq B$. We conclude that $w_{A}^{\prime} \geq w_{B}^{\prime} \geq w_{C}^{\prime}$. Hence, $u_{2}^{\prime}+w_{B}^{\prime}=4<5=v_{A}(\{2, B\})$ contradicting $\left(u^{\prime}, w^{\prime}\right) \in C\left(v_{A}\right)$. Hence, there is no pair $\left(u^{\prime}, w^{\prime}\right) \in C\left(v_{A}\right)$ with $u_{1}^{\prime}+w_{\tau(1)}^{\prime}=8, u_{2}^{\prime}+w_{\tau(2)}^{\prime}=4$, and $u_{3}^{\prime}+w_{\tau(3)}^{\prime}=2$ where $\tau:\{1,2,3\} \rightarrow M$ is a bijection with $w_{\tau(1)}^{\prime} \geq w_{\tau(2)}^{\prime} \geq w_{\tau(3)}^{\prime}$.

Our second result deals with a special case of object allocation situations. Let $\left(N, M, U, \sigma_{0}\right)$ be an object allocation situation where all agents prefer the first object over the second, the second object over the third, etc. Then, the object allocation game coincides with a corresponding permutation game.

Proposition 4.1 Let $\left(N, M, U, \sigma_{0}\right)$ be an object allocation situation with $U_{j 1} \geq \ldots \geq U_{m j}$ for all $j \in N$ and let $(N, v)$ be its corresponding object allocation game. Let $(N, M, U)$ be the corresponding permutation situation and $\left(N, v_{P}\right)$ its corresponding game. Then, the games $(N, v)$ and $\left(N, v_{P}\right)$ coincide.

Proof: We show that for all $S \subseteq N$ it holds that $v(S)=v_{P}(S)$. Let $S \subseteq N$ and consider the extensive form game $\left(\{S, N \backslash S\}, T, C^{S}, u^{S}\right)$. First we show, by giving a strategy for player $S$, that at the extensive form game player $S$ can obtain a payoff of at least $v_{P}(S)$. This implies $v(S) \geq v_{P}(S)$.

Consider the following strategy $z_{S} \in \Sigma_{S}$ for player $S$ at the extensive form game: "always pick the remaining object with lowest index number," i.e., the remaining object with highest utility. In other words, $z_{S}$ is such that $z_{S}(\sigma)=\tau$ for each $\sigma \in \mathcal{S}_{k}, k+1 \in S$, and $\tau \in \mathcal{S}_{k+1}$ with $\tau(k+1)=\min \{j: j \in M \backslash\{\sigma(1), \ldots, \sigma(k)\}\}$. Let the best reply of player $N \backslash S$ against this strategy of $S$ result in a set of objects $A=\left\{a_{1}, \ldots, a_{|S|}\right\} \subseteq M$ for $S$. We assume, without loss of generality, that the elements of $A$ are ordered $a_{1}<a_{2}<\ldots<a_{|S|}{ }^{4}$

Denote the set of objects initially owned by $S$ in the permutation situation $(N, M, U)$ by $B=$ $\left\{b_{1}, \ldots, b_{|S|}\right\}$. We assume, without loss of generality, that this set is ordered $b_{1}<b_{2}<\ldots<b_{|S|}$. Note that by definition of strategy $z_{S}$ player $S$ will obtain a better set of objects at the extensive form game in the sense that $a_{j} \leq b_{j}$ for all $j \in\{1, \ldots,|S|\}$. Now let $\pi^{*}: S \rightarrow B$ be the optimal reallocation of the objects in $B$ among the members of $S$, i.e., $\sum_{i \in S} U_{i \pi^{*}(i)}=\max \left\{\sum_{i \in S} U_{i \pi(i)}\right.$ : $\pi \in \Pi(S, B)\}$. Furthermore, define $\bar{\pi}: S \rightarrow A$ by $\bar{\pi}(i)=a_{j}$ if and only if $\pi^{*}(i)=b_{j}$. In other words, assign the $j$-th object of $A$ to player $i$ if and only if it is optimal to assign the $j$-th object of $B$ to $i$. Now

$$
\begin{aligned}
v_{P}(S)=\max \left\{\sum_{i \in S} U_{i \pi(i)}: \pi \in \Pi(S, B)\right\} & =\sum_{i \in S} U_{i \pi^{*}(i)} \\
& \leq \sum_{i \in S} U_{i \bar{\pi}(i)} \leq \max \left\{\sum_{i \in S} U_{i \pi(i)}: \pi \in \Pi(S, A)\right\} \leq v(S) .
\end{aligned}
$$

The first inequality holds because $\pi^{*}(i) \geq \bar{\pi}(i)$ for all $i \in S$. The second inequality is satisfied because $\bar{\pi}$ might be non-optimal. The last inequality is satisfied since the strategy of $S$ might be non-optimal.

\footnotetext{
${ }^{4}$ Recall that $M=\{1, \ldots, m\}$ and that $a_{i}<a_{k}$ implies that $U_{j i} \geq U_{j k}$ for all $j \in N$.
} 
Finally, we prove the inequality $v(S) \leq v_{P}(S)$ by considering the following strategy for $N \backslash S$ : "always pick the remaining object with lowest index number." It is obvious that if $N \backslash S$ uses this strategy, then $S$ cannot do better than to obtain the set of objects $B$. By reallocation of the objects in $B$ player $S$ obtains a maximal total utility of $v_{P}(S)$. Therefore, $v(S) \leq v_{P}(S)$.

\section{References}

[1] A. Mas-Colell, M. Whinston and J. Green, Microeconomic theory (Oxford University Press, Oxford, 1995).

[2] T. Quint, On one-sided versus two-sided matching markets, Games and Economic Behavior 16 (1996) 124-134.

[3] L. Shapley and H. Scarf, On cores and indivisibility, Journal of Mathematical Economics 1 (1974) $23-37$.

[4] L. Shapley and M. Shubik, The assignment game I: the core, International Journal of Game Theory 1 (1971) 111-130.

[5] L.-G. Svensson, Queue allocation of indivisible goods, Social Choice and Welfare 11 (1994) 323-330.

[6] S. Tijs, T. Parthasarathy, J. Potters and V. Rajendra Prasad, Permutation games: another class of totally balanced games, OR Spektrum 6 (1984) 119-123. 